\title{
Adaptive Self Recurrent Wavelet Neural Network and Sliding Mode Controller/Observer for a Slider Crank Mechanism
}

\author{
Ahmad Taher Azar \\ College of Engineering, Prince Sultan University, Riyadh, KSA \\ and \\ Faculty of Computers and Artificial Intelligence,Benha University, Egypt \\ E-mail: ahmad_t_azar@ieee.org; ahmad.azar@fci.bu.edu.eg \\ *Corresponding author
}

\section{Fernando E. Serrano}

Central American Technical University UNITEC,

Zona Jacaleapa, Tegucigalpa, Honduras.

E-mail: serranofer@eclipso.eu

\section{Josep M. Rossell}

Department of Mathematics, Polytechnic University of Catalonia, Barcelona, Spain

E-mail: josep.maria.rossell@upc.edu

\section{Sundarapandian Vaidyanathan}

Research and Development Centre, Vel Tech University, Chennai, India.

E-mail: sundar@veltech.edu.in

\section{Quanmin Zhu}

Department of Engineering Design and Mathematics, University of the West of England, Bristol, UK.

E-mail: quan.zhu@uwe.ac.uk

\begin{abstract}
In this paper, a novel control strategy based on an adaptive self recurrent wavelet neural network (SRWNN) and a sliding mode controller/observer for a slider crank mechanism is proposed. The aim is to reduce the tracking error of the linear displacement of this mechanism while following a specified trajectory. The controller design consists of two parts. The first one is a sliding mode control strategy and the second part is an adaptive self recurrent wavelet neural network (SRWNN) controller. This controller is trained offline first, and then the SRWNN weights are updated online by the adaptive control law. Apart from the hybrid control strategy proposed in this paper, a velocity observer is implemented to replace the use of velocity sensors. This study begins with the derivation of the dynamic equations of the slider crank mechanism followed by the derivation of the proposed observer and control strategies. Finally, to demonstrate the effectiveness of the controller/observer strategy, a numerical example is supplied to analyze the variables of the system, tracking error and the estimated variables.
\end{abstract}

Keywords: Adaptive wavelet neural networks; Sliding mode control; Sliding mode observer; Slider crank mechanism

\section{Introduction}

The slider crank is a mechanism with several applications in industry and other fields such as gasoline and diesel engines (Wai, 2003), where the linear displacement generated by a force is converted to a circular movement or vice versa (Haddad and Chellaboina, 2002; Lin et al, 1999). Even when in the literature some traditional 
approaches are used (Komaita and Furuta, 2008; Saito et al, 2009; Wai, 2003), the main objective of this study is to improve the previously mentioned works. As it is known, wavelet neural networks are implemented in different applications as shown in Alexandridis and Zapranis (2013); Min et al (2014); Yoo et al (2007, 2005, 2006). In this paper, a novel approach is presented considering these control strategies. The main idea is to develop and design an appropriate control methodology taking into account a hybrid strategy which consists of a sliding mode controller (Azar and Serrano, 2015, 2018; Azar and Zhu, 2015; Vaidyanathan and Azar, 2015; Vaidyanathan et al, 2015) together with and adaptive self recurrent wavelet neural networks (SRWNN) (Abiyev et al, 2013; Hongyan et al, 2010, 2007; Wu et al, 2014; Yoo et al, 2007; Taha and Taha, 2018) to stabilize this mechanical system. Note that a slider crank mechanism is a closed chain mechanical system. There are many examples of observers for nonlinear systems that are fundamental for the proposed observer shown in this paper, such as, Davila et al (2005); Kalsi et al (2009); Su et al (2007). In this paper, a full order observer is presented to obtain the estimated velocities without the need of sensors (Wai, 2003; Wang et al, 2014; Xiao and Zhu, 2014). Considering the work of Hongyan et al $(2010,2007)$ an adaptive self recurrent wavelet neural network and sliding mode controller for the slider crank mechanism is proposed. The reason to implement a self recurrent wavelet neural network and sliding mode controller is that this control strategy has been demonstrated to be superior to other neural network controller approaches. By implementing the separation principle, the observer and controller can be designed independently (Alexandridis and Zapranis, 2013; Lin et al, 1999; Sawada and Itamiya, 2012; Yoo et al, 2005). The self recurrent wavelet neural network controller architecture consists of four layers: the input layer, a mother wavelet layer, a product layer and an output layer (Yoo et al, 2005) which makes this controller part more efficient than other neural network controller approaches.

\section{Dynamic model of the slider crank mechanism}

In this section, the dynamic model of the slider crank mechanism is presented. A complete dynamic model based on the Euler-Lagrange equations is derived considering all the system states that mark a complete difference from other dynamical models found in literature (Haddad and Chellaboina, 2002; Lin et al, 1999; Saito et al, 2009). In Fig. 1, the slider crank mechanism is depicted. As can be noticed, the linear motion is converted to rotational motion by applying a force $F_{1}(t)$. This produces a linear motion $a(t)$ that generates a rotational movement on each joint $\theta_{1}(t)$,

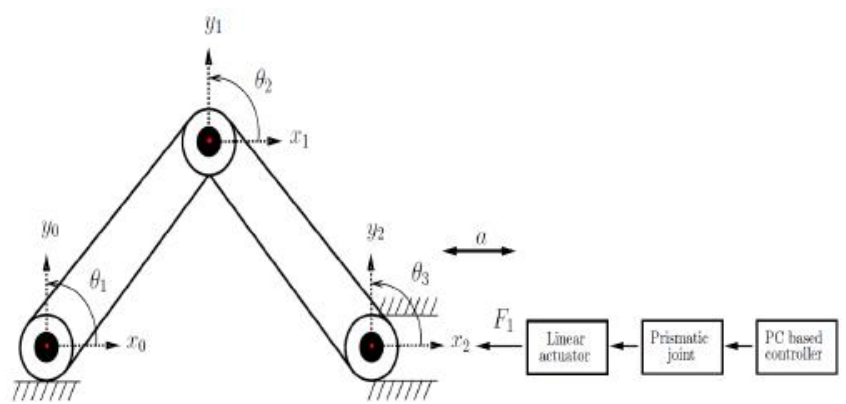

Figure 1 Slider crank mechanism

$\theta_{2}(t)$ and $\theta_{3}(t)$. The Euler-Lagrange equations are given by:

$$
D(q(t)) \ddot{q}(t)+C(q(t), \dot{q}(t)) \dot{q}(t)+g(q(t))=\tau(t),
$$

where $q(t) \in \Re^{n}$ is the angular position and linear displacement vector, $\tau \in \Re^{n}$ is the torque/force vector, $D \in \Re^{n \times n}$ is the positive definite inertia matrix, $C \in$ $\Re^{n \times n}$ is the torque vector due to Coriolis and centrifugal forces and $g \in \Re^{n}$ is the torque vector yielded by gravitational forces. The dimension of the slider crank mechanical system is $n=4$, where the vectors $q(t)$ and $\tau(t)$ are defined by:

$$
q(t)=\left[\begin{array}{c}
\theta_{1}(t) \\
\theta_{2}(t) \\
\theta_{3}(t) \\
a
\end{array}\right], \tau(t)=\left[\begin{array}{c}
0 \\
0 \\
0 \\
F_{1}(t)
\end{array}\right]
$$

In order to implement the proposed control strategy, the dynamic equation (1) is divided into the following individual parts (Spong et al, 2006):

$$
\begin{gathered}
\sum_{j=1}^{n} D_{k j}(q(t)) \ddot{q}_{j}(t) \\
+\sum_{i=1}^{n} \sum_{j=1}^{n} C_{i j k}(q(t), \dot{q}(t)) \dot{q}_{i}(t) \dot{q}_{j}(t)+g_{k}(q(t))=\tau_{k}(t),
\end{gathered}
$$

where $D_{k j}(q(t))$ is the $(j, k)$ element of the inertia matrix, $C_{i j k}(q(t), \dot{q}(t))$ is the $(i, j)$ Christoffel symbol, $g_{k}(q(t))$ is the $k$ element of the gravity vector and $\tau_{k}(t)$ is the $k$ element of the input vector $\tau(t)$, where $k=1,2,3,4$ for the slider crank dynamic equation. The components of the inertia matrix are explained in the Appendix. For convenience, the $j$ element of the angular/linear position vector and force/torque vector are given by $q_{j}(t)$ and $\tau_{j}(t)$ respectively.

\section{Control system architecture and Observer design}

The control system architecture for the slider crank mechanism is depicted in Fig. 2. This control strategy 
is based on simulation but the hardware and actuator dynamics can be added with a force to current converter (Tahmasebi et al, 2014). The proposed adaptive controller technique is formed by the following three components: (1) the adaptive self recurrent wavelet neural network controller; (2) sliding mode controller and (3) a full order nonlinear observer.

The implemented observer is based on different observer approaches for several kinds of mechanical systems. The observer is based on Davila et al (2005); $\mathrm{Su}$ et al (2007); De Wit and Fixot (1991), where the inverse dynamics of an $n$ degrees of freedom mechanical system is implemented along with other techniques such as sliding mode to develop a successful observer. Examples of sliding mode observers for nonlinear systems can be found in Kalsi et al (2009); Xiao and Zhu (2014).

In order to design a suitable observer to estimate the state variables of the slider crank mechanism, the first step is to convert the dynamic model (1) of the slider crank mechanism to state space by selecting the following state variables:

$$
x_{1}(t)=q(t), x_{2}(t)=\dot{q}(t)
$$

obtaining the state space model

$$
\begin{aligned}
& \dot{x}_{1}(t)=x_{2}(t) \\
& \dot{x}_{2}(t)=D^{-1}\left(x_{1}(t)\right)\left[\tau(t)-C\left(x_{1}(t), x_{2}(t)\right) x_{2}(t)-g\left(x_{1}(t)\right)\right] .
\end{aligned}
$$

Therefore, the state space representation (5) of the slider crank mechanism is:

$$
\begin{gathered}
\dot{x}_{1}(t)=x_{2}(t) \\
\dot{x}_{2}(t)=f\left(t, x_{1}(t), x_{2}(t), \tau(t)\right) .
\end{gathered}
$$

Considering that all the states of the slider crank mechanism will be estimated from the measured position $x_{1}(t)$, the following observer is proposed to estimate all the state variables of this mechanism:

$$
\begin{aligned}
& \dot{\hat{x}}_{1}(t)=\hat{x}_{2}(t) \\
& \dot{\hat{x}}_{2}(t)=-k_{0} \operatorname{sgn}(\mathrm{e}(\mathrm{t}))+f\left(t, x_{1}(t), \hat{x}_{2}(t), \tau(t)\right)-k_{1} e(t),
\end{aligned}
$$

where $\hat{x}_{1}(t)$ and $\hat{x}_{2}(t)$ are the estimated position and velocity vectors, $k_{0}$ and $k_{1}$ are positive constants and $\operatorname{sgn}(\cdot)$ is the sign function. The error signals $e(t)$ and $\dot{e}(t)$ are defined by:

$$
\begin{aligned}
& e(t)=\hat{x}_{1}(t)-x_{1}(t) \\
& \dot{e}(t)=\hat{x}_{2}(t)-\dot{x}_{1}(t)=\hat{x}_{2}(t)-x_{2}(t) .
\end{aligned}
$$

These are the error signals implemented in (7) and they are used in the development of the error dynamics of the system employed in the convergence test of the estimated variables. Therefore, the system error dynamics is given by Su et al (2007); De Wit and Fixot (1991)

$$
\begin{aligned}
\ddot{e}(t) & =\dot{\hat{x}}_{2}(t)-\dot{x}_{2}(t)= \\
& -k_{0} \operatorname{sgn}(\mathrm{e}(\mathrm{t}))+f\left(t, x_{1}(t), \hat{x}_{2}(t), \tau(t)\right) \\
& -k_{1} e(t)-f\left(t, x_{1}(t), x_{2}(t), \tau(t)\right) .
\end{aligned}
$$

Now, defining the variable

$$
r(t)=\dot{e}(t)+e(t)
$$

The dynamic errors defined by the signal $r(t)$ is:

$$
\begin{aligned}
\dot{r}(t)=- & k_{0} \operatorname{sgn}(\mathrm{e}(\mathrm{t}))+f\left(t, x_{1}(t), \hat{x}_{2}(t), \tau(t)\right)-k_{1} e(t) \\
& -f\left(t, x_{1}(t), x_{2}(t), \tau(t)\right)+\hat{x}_{2}(t)-x_{2}(t) .
\end{aligned}
$$

The error equation (11) is important because the convergence of the observer explained in this section can be corroborated.

Theorem 1: Consider the observer obtained in (7). If $e(t)=0$ as $t \rightarrow \infty$, then the asymptotic convergence of the state variables is assured.

Proof: This condition is tested by selecting an appropriate Lyapunov functional $V(r(t), e(t))$ to prove that $\dot{V}(r(t), e(t)) \leq 0$. Consider the following Lyapunov functional:

$V(r(t), e(t))=\frac{1}{2} r^{T}(t) r(t)+\frac{1}{2} e^{T}(t) e(t)$.

Now, deriving (12) we obtain

$\dot{V}(r(t), e(t))=r^{T}(t) \dot{r}(t)+e^{T}(t) \dot{e}(t)$.

Substituting the derivatives of the error signals $\dot{r}(t)$ and $\dot{e}(t)$ from (11) and (8), respectively, yields:

$$
\begin{aligned}
\dot{V}(r(t), e(t)) & =-r^{T}(t) k_{0} \operatorname{sgn}(\mathrm{e}(\mathrm{t})) \\
& +r^{T}(t) f\left(t, x_{1}(t), \hat{x}_{2}(t), \tau(t)\right)-r^{T}(t) k_{1} e(t) \\
& -r^{T}(t) f\left(t, x_{1}(t), x_{2}(t), \tau(t)\right)+r^{T}(t) \hat{x}_{2}(t) \\
& -r^{T}(t) x_{2}(t)+e^{T}(t) \hat{x}_{2}(t)-e^{T}(t) x_{2}(t) .
\end{aligned}
$$

Rearranging (14) we have

$$
\begin{aligned}
\dot{V}(r(t), e(t)) & =-r^{T}(t) k_{0} \operatorname{sgn}(\mathrm{e}(\mathrm{t})) \\
& -r^{T}(t)\left(f\left(t, x_{1}(t), x_{2}(t), \tau(t)\right)\right. \\
& \left.-f\left(t, x_{1}(t), \hat{x}_{2}(t), \tau(t)\right)\right)-r^{T}(t) k_{1} e(t) \\
& -r^{T}(t)\left(x_{2}(t)-\hat{x}_{2}(t)\right) \\
& -e^{T}(t)\left(x_{2}(t)-\hat{x}_{2}(t)\right) .
\end{aligned}
$$

Then, an upper bound for the derivative of the Lyapunov function is found in the following form (Liu, 1999; Xiang and Siow, 2004):

$$
\begin{array}{r}
\dot{V}(r(t), e(t)) \leq-k_{0 \min }\left\|r^{T}(t) \operatorname{sgn}(\mathrm{e}(\mathrm{t}))\right\| \\
-k_{1 \min }\left\|r^{T}(t) e(t)\right\| \\
-\left\|r^{T}(t)\right\|\left\|f\left(t, x_{1}(t), x_{2}(t), \tau(t)\right)-f\left(t, x_{1}(t), \hat{x}_{2}(t), \tau\right)\right\| \\
-\left\|r^{T}(t)\right\|\left\|x_{2}(t)-\hat{x}_{2}(t)\right\|-\left\|e^{T}(t)\right\|\left\|x_{2}(t)-\hat{x}_{2}(t)\right\|,
\end{array}
$$




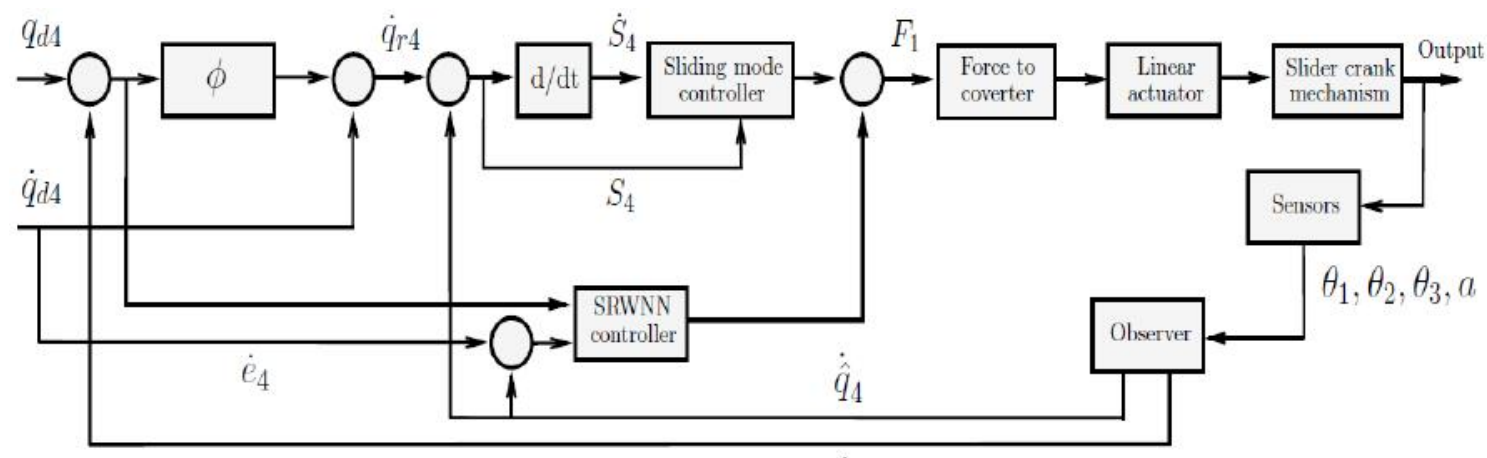

$\hat{q}_{4}$

Figure 2 Control system architecture

where

$$
k_{0 \min }, k_{1 \min }>0, k_{0 \min }=\min _{i \in n} k_{0 i}, k_{1 \min }=\min _{i \in n} k_{1 i},
$$

Thus, the global asymptotic convergence of the observer is ensured and the proof is completed.

\section{Controller design}

In this section, the design and development of an adaptive self recurrent wavelet neural network and sliding mode controller is described. As shown in Fig. 3 , the controller architecture consists of a sliding mode and an adaptive SRWNN controller in which their input signals are determined by the error signals $e(t), \dot{e}(t)$, that are used as the inputs of the controller, and $S, \dot{S}$ as the sliding variables.

\subsection{Self recurrent wavelet neural network controller structure and offline training}

The inputs of the SRWNN controller part are established by the dynamic errors of the slider crank mechanism that, in this case, are the linear displacement error $e_{4}(t)$ and the linear velocity error $\dot{e}_{4}(t)$. A schematic diagram of the self recurrent wavelet neural network controller is shown in Fig. 3. The hybrid control strategy consists of the following four layers as defined in (Alexandridis and Zapranis, 2013; Yoo et al, 2005, 2006):

Layer 1: The input layer of the SRWNN controller part with inputs $e_{4}(t)$ and $\dot{e}_{4}(t)$.

Layer 2: The mother wavelet layer in which each node is a mother wavelet with a self-feedback loop. In this article a function similar to $\phi(x(t))=-x(t) \exp \left(-\frac{1}{2} x^{2}(t)\right)$ is implemented as a mother wavelet function.

Layer 3: The product layer, where the product of the mother wavelets is obtained.

Layer 4: The output layer, where the sum of the products obtained in layer 3 and the weighted inputs are computed.

The neural network architecture for the SRWNN controller part of the slider crank mechanism is designed

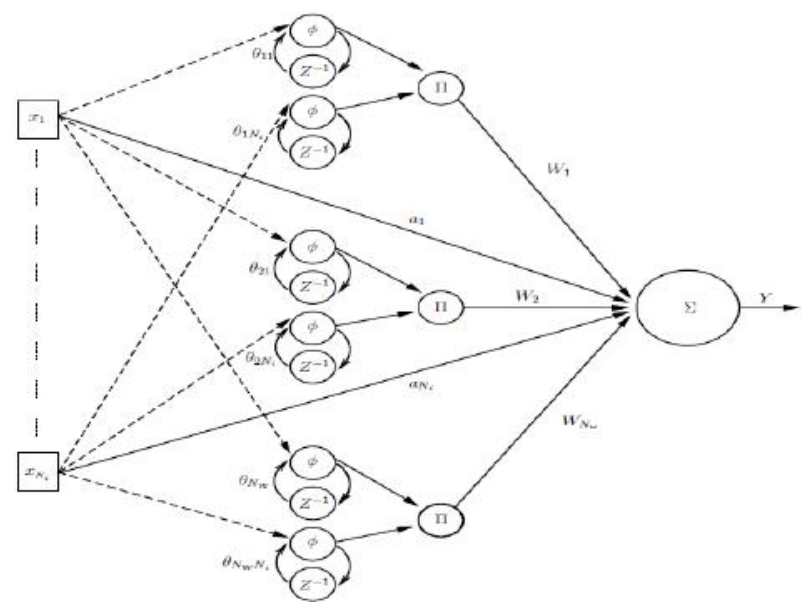

Figure 3 SRWNN controller architecture

with two input signals, the linear displacement error $e_{4}(t)$ and the linear velocity error $\dot{e}_{4}(t)$. The mother wavelets of the second layer are defined by:

$$
\begin{gathered}
\phi\left(z_{j k}(n)\right)= \\
-\left(\frac{u_{j k}(n)-m_{j k}}{d_{j k}}\right) \exp \left(-\frac{1}{2}\left(\frac{u_{j k}(n)-m_{j k}}{d_{j k}}\right)^{2}\right),
\end{gathered}
$$

where

$$
\phi\left(z_{j k}(n)\right)=\phi\left(\frac{u_{j k}(n)-m_{j k}}{d_{j k}}\right),
$$

with

$$
z_{j k}(n)=\frac{u_{j k}(n)-m_{j k}}{d_{j k}},
$$

where $m_{j k}$ and $d_{j k}$ are the translation and dilation factors of the mother wavelet (Yoo et al, 2005, 2006). The subscript $j, k$ is the $k$ input term of the $j$ wavelet. The inputs of this layer, in discrete time $n$, are defined by:

$$
u_{j k}(n)=x_{k}(n)+\phi_{j k}(n-1) \theta_{j k},
$$


where $\theta_{j k}$ is the weight of the auto-feedback loop shown in Fig. 3 due to the feedback in each mother wavelet in layer 2. In layer 3 , the product of each mother wavelet or wavelon is denoted by:

$$
\begin{gathered}
\Phi_{j}(x(n))=\prod_{k=1}^{N_{i}} \phi\left(z_{j k}(n)\right)= \\
\prod_{k=1}^{N_{i}}\left[-\left(\frac{u_{j k}(n)-m_{j k}}{d_{j k}}\right) \exp \left(-\frac{1}{2}\left(\frac{u_{j k}(n)-m_{j k}}{d_{j k}}\right)^{2}\right)\right] .
\end{gathered}
$$

The signal provided by the output layer is given by:

$$
y(n)=\sum_{j=1}^{N_{w}} w_{j} \Phi_{j}(x(n))+\sum_{k=1}^{N_{i}} a_{k} x_{k}(n),
$$

where $w_{j}$ is the connection weight between the product node and the output node, and $a_{k}$ is the connection weight between the input node and the output node. In order to compute the weights of the adaptive SRWNN controller for initialization purpose, a weight vector $W$ is defined as:

$$
W=\left[a_{k}, m_{j k}, d_{j k}, \theta_{j k}, w_{j}\right]^{T},
$$

where the weights explained in this subsection are grouped into one vector in order to train the adaptive SRWNN controller part offline before the real time implementation.

\subsection{Offline Training}

The offline training of the SRWNN controller part of this strategy is crucial for a suitable initialization of the adaptive SRWNN weight vector. The training method implemented for this purpose is the well-known gradient descent method (Alexandridis and Zapranis, 2013; Yoo et al, 2007, 2005, 2006) which consists of the minimization of an objective function as described below.

$$
J(n)=\frac{1}{2}\left[y_{d}(n)-y(n)\right]^{2}=\frac{1}{2} e^{2}(n),
$$

where $y_{d}(n)$ is the desired output and $y(n)=\hat{y}_{d}(n)$ is the actual output of the adaptive SRWNN controller part. In this method the weight vector $W$ is updated in each iteration while minimizing the functional cost (25). It is defined in (Yoo et al, 2007) as:

$$
W(n+1)=W(n)+\Delta W(n)=W(n)+\bar{\eta}\left(-\frac{\partial J(n)}{\partial W(n)}\right),
$$

where

$$
\bar{\eta}=\operatorname{diag}\left[\eta^{a}, \eta^{m}, \eta^{d}, \eta^{\theta}, \eta^{w}\right]
$$

and the partial derivative of the functional cost is given by:

$$
\frac{\partial J(n)}{\partial W(n)}=-e(n) \frac{\partial y(n)}{\partial W(n)} .
$$

Applying the chain rule recursively, the adjustments of the weights of each layer can be obtained. The components of the weight vector are given by:

$$
\frac{\partial y(n)}{\partial a_{k}(n)}=x_{k}(n)
$$

$$
\frac{\partial y(n)}{\partial m_{j k}(n)}=-\frac{w_{j}}{d_{j k}} \frac{\partial \Phi_{j}(x(n))}{\partial z_{j k}(n)}
$$

$$
\frac{\partial y(n)}{\partial d_{j k}(n)}=-\frac{w_{j}}{d_{j k}} z_{j k}(n) \frac{\partial \Phi_{j}(x(n))}{\partial z_{j k}(n)}
$$

$$
\begin{aligned}
\frac{\partial y(n)}{\partial \theta_{j k}(n)} & =\frac{w_{j}}{d_{j k}} \phi_{j k}(n-1) \frac{\partial \Phi_{j}(x(n))}{\partial z_{j k}(n)}, \\
\frac{\partial y(n)}{\partial w_{j}(n)} & =\Phi_{j}(x(n)),
\end{aligned}
$$

where

$$
\frac{\partial \Phi_{j}(x(n))}{\partial z_{j k}(n)}=\left(z_{j k}^{2}(n)-1\right) \exp \left(-\left(\frac{1}{2}\right) z_{j k}^{2}(n)\right) .
$$

With these partial derivatives the SRWNN controller can be trained by this iterative algorithm to initialize the weight vector of the adaptive SRWNN control. 


\subsection{Adaptive SRWNN and Sliding Mode Controller Design}

In order to derive the necessary equations with their respective stability conditions for the control of the linear displacement of the slider crank mechanism, consider the following hybrid control law (Hongyan et al, 2007):

$$
\tau_{4}(t)=F_{1}(t)=U_{s m c}(t)+U_{N N}(t),
$$

where

$$
U_{s m c}(t)=k_{a} \operatorname{sgn}\left(\dot{s}_{4}(t)\right)+k_{b} s_{4}(t)
$$

is the sliding mode controller part of the overall controller and $k_{a}, k_{b}>0$ are the sliding gains, $\dot{s}_{4}$ and $s_{4}$ the derivative and original variables for the dynamic errors of the slider crank mechanism defined later. Moreover,

$$
U_{N N}(t)=\sum_{j=1}^{N_{w}} w_{j} \Phi_{j}(x(n))+\sum_{k=1}^{N_{i}} a_{k} x_{k}(n)
$$

is the adaptive self recurrent wavelet neural network controller part of the overall controller where its weights have been defined previously with only one SRWNN controller output. To design the controller, it is necessary to include the Coriolis matrix components shown in (3).

$$
C_{k, j}(q(t), \dot{q}(t))=\sum_{i=1}^{4} C_{i j k}(q(t)) \dot{q}_{i}(t),
$$

where $j, k=1,2,3,4$. Define the terms of the position vector $(2)$ as $q_{j}(t)$, where $q_{1}(t)=\theta_{1}(t), q_{2}(t)=\theta_{2}(t)$, $q_{3}(t)=\theta_{3}(t)$ and $q_{4}(t)=a(t)$ are the respective angular positions and linear displacements of the slider crank mechanism, considering that there is only one input (the force $\left.\tau_{4}(t)=F_{1}(t)\right)$. The following error signals:

$$
e_{j}(t)=q_{d j}(t)-q_{j}(t)
$$

are necessary for the development of the proposed hybrid control strategy and to test the stability of the system, where $q_{d j}(t)$ is the desired reference trajectory for the $j$ joint (Liu, 1999; Slotine and Weiping, 1988; Xiang and Siow, 2004).

$$
\begin{aligned}
& \dot{q}_{r j}(t)=\dot{q}_{d j}(t)+\Phi e_{j}(t), \\
& s_{j}(t)=\dot{q}_{r j}(t)-\dot{q}_{j}(t),
\end{aligned}
$$

and

$$
\dot{s}_{j}(t)=\ddot{q}_{r j}(t)-\ddot{q}_{j}(t) .
$$

Due to the convergence of the dynamic errors of the observer, the following assumptions are implemented:

$$
\begin{gathered}
e_{j}(t) \rightarrow 0, \dot{e}_{j}(t) \rightarrow 0, \ddot{e}_{j}(t) \rightarrow 0 \\
q_{j}(t) \simeq \hat{q}_{j}(t), \dot{q}_{j}(t) \simeq \dot{\hat{q}}_{j}(t), \ddot{q}_{j}(t) \simeq \ddot{\hat{q}}_{j}(t) .
\end{gathered}
$$

With these considerations, the stability of the overall system (controller, observer and slider crank mechanism) is assured by the following theorem.

Theorem 2: $\quad$ The proposed control law (35) stabilizes the linear displacement position of the slider crank mechanism.

Proof: This condition is tested by selecting an appropriate Lyapunov functional $V(s)$ and proving that $\dot{V}(s) \leq 0$ assuring the global asymptotic stability of the overall control system, so $q_{4}(t)$ is stabilized when $t \rightarrow \infty$. In order to prove the theorem, it is necessary to define the dynamic errors of the system as follows (Seraji, 1989):

$$
\begin{gathered}
D_{44}(q(t)) \dot{s}_{4}(t) \\
+\sum_{\substack{j=1 \\
j \neq 4}}^{n} D_{4 j}(q(t)) \dot{s}_{j}(t)+\sum_{j=1}^{n} C_{4 j}(q(t), \dot{q}(t)) s_{j}(t)= \\
-F_{1}(t)+D_{44}(q(t))\left(\ddot{q}_{d 4}(t)+\Phi \dot{e}_{4}(t)\right) \\
+\sum_{\substack{j=1 \\
j \neq 4}}^{n} D_{4 j}(q(t))\left(\ddot{q}_{d j}(t)+\Phi \dot{e}_{j}(t)\right) \\
+\sum_{j=1}^{n} C_{4 j}(q(t), \dot{q}(t))\left(\dot{q}_{d j}(t)+\Phi e_{j}(t)\right)+g_{4}(q(t)),
\end{gathered}
$$

for $n=4$, where $D_{i j}$ is the $(i, j)$ element of the inertia matrix, $C_{i j}$ is the $(i, j)$ element of the Coriolis matrix and $g_{4}(q(t))$ is the fourth element of the gravity vector. Considering that $D_{41}(q(t))=D_{42}(q(t))=D_{43}(q(t))=0$ (see Appendix), (44) becomes

$$
\begin{array}{r}
D_{44}(q(t)) \dot{s}_{4}(t)+\sum_{j=1}^{n} C_{4 j}(q(t), \dot{q}(t)) s_{j}(t)= \\
-F_{1}(t)+D_{44}(q(t))\left(\ddot{q}_{d 4}(t)+\Phi \dot{e}_{4}(t)\right) \\
+\sum_{j=1}^{n} C_{4 j}(q(t), \dot{q}(t))\left(\dot{q}_{d j}(t)+\Phi e_{j}(t)\right)+g_{4}(q(t)) .
\end{array}
$$


Then, rearranging the terms in (45) and substituting (40) and its derivative, we obtain:

$$
\begin{aligned}
D_{44}(q(t)) \dot{s}_{4}(t) & =-F_{1}(t)-\sum_{j=1}^{n} C_{4 j}(q(t), \dot{q}(t)) s_{j}(t) \\
& +D_{44}(q(t)) \ddot{q}_{r 4}(t) \\
& +\sum_{j=1}^{n} C_{4 j}(q(t), \dot{q}(t)) \dot{q}_{r j}(t)+g_{4}(q(t)) .
\end{aligned}
$$

Now, considering the linear parameterization of the mechanical system as shown in (Slotine and Weiping, 1988), the following equation is obtained:

$$
\begin{gathered}
D_{44}(q(t)) \ddot{q}_{r 4}(t)+\sum_{j=1}^{n} C_{4 j}(q(t), \dot{q}(t)) \dot{q}_{r j}(t)+q_{4}(q(t)) \\
=Y_{4}\left(q(t), \dot{q}(t), \dot{q}_{r j}(t), \ddot{q}_{r j}(t)\right) \widetilde{W}
\end{gathered}
$$

where $Y_{4}$ is the fourth row of the dynamic parameters of the system and $\widetilde{W}$ is the parameter estimation error. By implementing (47) in (46) and rearranging the terms, yields

$$
\begin{aligned}
\dot{s}_{4}(t) & =-D_{44}^{-1}(q(t)) F_{1}(t) \\
& -D_{44}^{-1}(q(t)) \sum_{j=1}^{n} C_{4 j}(q(t), \dot{q}(t)) s_{j}(t) \\
& +D_{44}^{-1}(q(t)) Y_{4}\left(q(t), \dot{q}(t), \dot{q}_{r j}(t), \ddot{q}_{r j}(t)\right) \widetilde{W} .
\end{aligned}
$$

Now, the following Lyapunov function to test the stability of the overall system is considered:

$$
V(s(t))=\frac{1}{2} s_{4}^{2}(t)+\frac{1}{2} \widetilde{W}^{T} \widetilde{W} .
$$

Deriving (49) yields

$$
\dot{V}(s(t))=s_{4}(t) \dot{s}_{4}(t)+\dot{\widetilde{W}}^{T} \widetilde{W} .
$$

Substituting (48) into (50), we obtain

$$
\begin{aligned}
\dot{V}(s(t)) & =-s_{4}(t) D_{44}^{-1}(q(t)) F_{1}(t) \\
& -s_{4}(t) D_{44}^{-1}(q(t)) \sum_{j=1}^{n} C_{4 j}(q(t), \dot{q}(t)) s_{j}(t) \\
& +s_{4}(t) D_{44}^{-1}(q(t)) Y_{4}\left(q(t), \dot{q}(t), \dot{q}_{r j}(t), \ddot{q}_{r j}(t)\right) \widetilde{W} \\
& +\dot{\widetilde{W}}^{T} \widetilde{W} .
\end{aligned}
$$

The adaptive laws for the SRWNN controller part of the overall controller is:

$$
\dot{\widetilde{W}}^{T}=-s_{4}(t) D_{44}^{-1}(q(t)) Y_{4}\left(q(t), \dot{q}(t), \dot{q}_{r j}(t), \ddot{q}_{r j}(t)\right) \widetilde{W} .
$$

Then,

$$
\begin{aligned}
\dot{V}(s(t)) & =-s_{4}(t) D_{44}^{-1}(q(t)) F_{1}(t) \\
& -s_{4}(t) D_{44}^{-1}(q(t)) \sum_{j=1}^{n} C_{4 j}(q(t), \dot{q}(t)) s_{j}(t) .
\end{aligned}
$$

The upper limit of the Lyapunov functional derivative is:

$$
\begin{aligned}
\dot{V}(s(t)) & \leq-\left\|s_{4}(t) D_{44}^{-1}(q(t)) F_{1}(t)\right\| \\
& -\sum_{j=1}^{n}\left\|s_{4}(t) D_{44}^{-1}(q(t)) C_{4 j}(q(t), \dot{q}(t)) s_{j}(t)\right\|,
\end{aligned}
$$

due to

$$
\begin{aligned}
& \Phi \leq \Lambda \\
& \Phi=\left\|s_{4}(t) D_{44}^{-1}(q(t)) \sum_{j=1}^{n} C_{4 j}(q(t), \dot{q}(t)) s_{j}(t)\right\| \\
& \Lambda=\sum_{j=1}^{n}\left\|s_{4}(t) D_{44}^{-1}(q(t)) C_{4 j}(q(t), \dot{q}(t)) s_{j}(t)\right\| .
\end{aligned}
$$

With the derivative of the Lyapunov function as shown in (54), the global asymptotic stability of the system is assured and the proposed hybrid controller strategy is suitable for the control of the slider crank mechanism.

\section{Numerical Simulations}

The simulation was performed in $M A T L A B{ }^{\circledR} 2018 \mathrm{~b}$ and SIMULINK ${ }^{\circledR}$ using an ODE45 solver. The objective of this simulation is to test the proposed controller/observer strategy implemented in the studied mechanism for position tracking of $q_{4}$ (estimating the velocities) and to corroborate that the tracking error is smaller than the obtained by other control strategies. The desired trajectory for the linear displacement $q_{4}$ is $q_{d 4}=0.3 \sin (2.5133 t)+0.3$ with the slider crank parameters shown in Table 1. A self recurrent wavelet neural network controller and a variation of the computed torque algorithm as shown in Sawada and Itamiya (2012); Wai (2003) are simulated for comparison purposes.

The parameters of the full order observer are:

$$
k_{0}=1 \times 10^{-5}\left[\begin{array}{cccc}
1 & 0 & 0 & 0 \\
0 & 1 & 0 & 0 \\
0 & 0 & 1 & 0 \\
0 & 0 & 0 & 1
\end{array}\right],
$$


Table 1 Slider crank mechanism parameters

\begin{tabular}{cccc}
\hline Link & $\begin{array}{c}\text { Length } \\
(m)\end{array}$ & $\begin{array}{c}\text { Center of mass } \\
(m)\end{array}$ & $\begin{array}{c}\text { Inertia tensor } \\
\left(\mathrm{Kg} \cdot \mathrm{m}^{2}\right)\end{array}$ \\
\hline 1 & 0.3 & 0.15 & 0.18 \\
2 & 0.5 & 0.25 & 0.18 \\
3 & 0.10 & 0.05 & 0.18 \\
\hline
\end{tabular}

and

$$
k_{1}=1 \times 10^{-5}\left[\begin{array}{cccc}
1 & 0 & 0 & 0 \\
0 & 1 & 0 & 0 \\
0 & 0 & 1 & 0 \\
0 & 0 & 0 & 1
\end{array}\right],
$$

while the sliding mode controller gains are $k_{a}=0.01$ and $k_{b}=0.01$. The neural network architecture of the adaptive SRWNN consists of two inputs $e_{4}(t)$ and $\dot{e}_{4}(t)$ with four mother wavelets or wavelons in layer 2 . The initial weights calculated by the gradient descent method are:

$$
\begin{aligned}
a_{k} & =[0.1999,3.5000], \\
m_{j k} & =[3.7000,1.3000,7.1000,6.0000], \\
d_{j k} & =[0.7000,1.8999,0.1500,6.4000], \\
\theta_{j k} & =[12.7000,10.4000,5.5000,0.9000], \\
w_{j} & =[2.3000,1.2000] .
\end{aligned}
$$

In Fig. 4 the linear displacement error $q_{d 4}(t)-q_{4}(t)$ is shown for the proposed and other control strategies. We can observe that the proposed hybrid controller has the smallest tracking error in comparison with (Sawada and Itamiya, 2012; Wai, 2003) considering that the accuracy is improved and the inverse dynamic is estimated by the SRWNN controller.

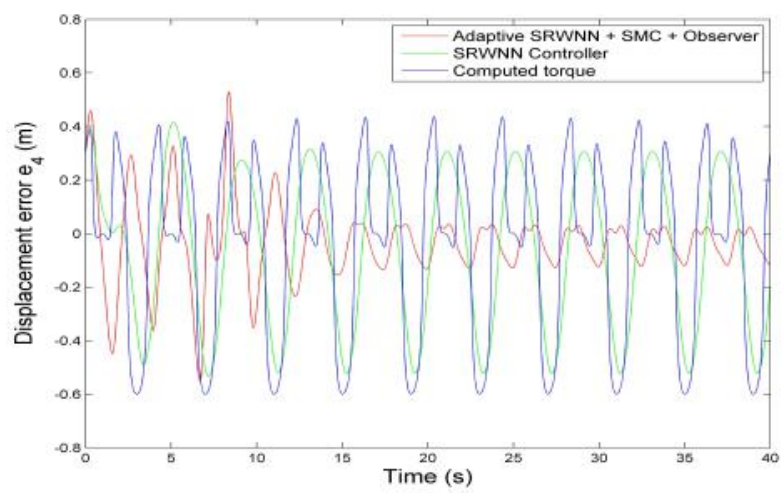

Figure 4 Tracking error for the linear displacement $q_{4}(t)$

In Fig. 5, the linear displacement $q_{4}(t)$ is depicted for the three control strategies, proving that the hybrid SRWNN control technique improves the position accuracy in comparison with (Sawada and Itamiya, 2012; Wai, 2003).

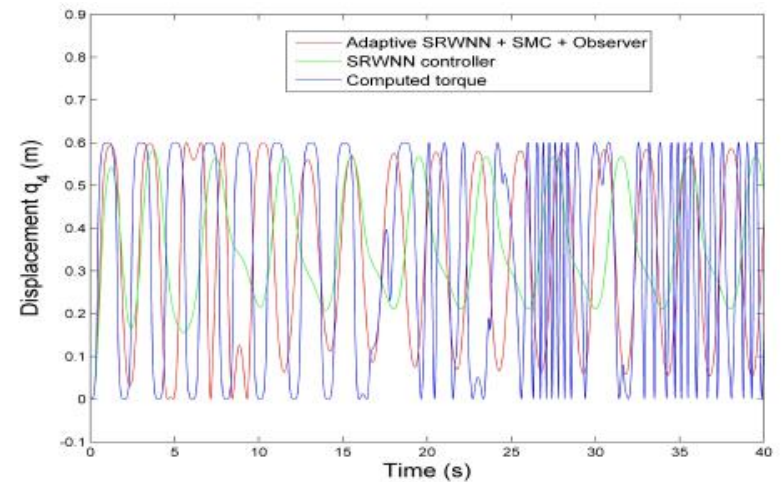

Figure 5 Linear displacement $q_{4}(t)$

In Fig. 6 the input forces $F_{1}(t)$ for the proposed and other control approaches are shown, which demonstrates that the smallest control effort is obtained with the proposed control technique in comparison with the other two strategies, avoiding the actuator saturation.

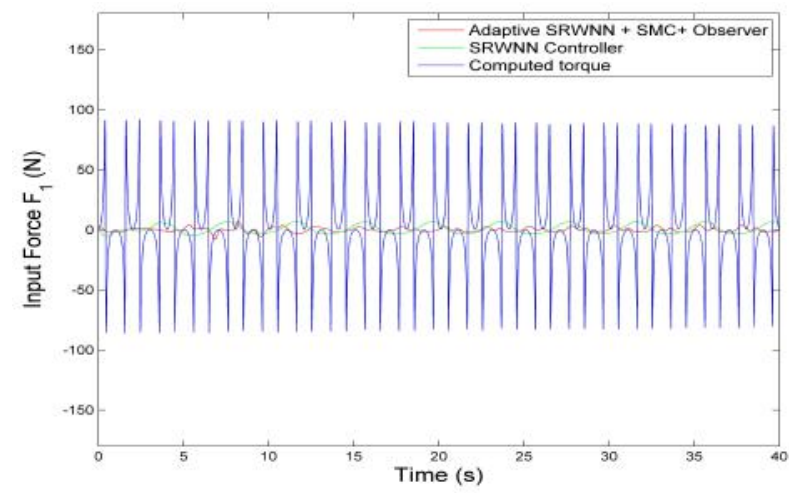

Figure 6 Input force $F_{1}(t)$

Finally, in Fig. 7 the angular displacement of the rotational arm $\theta_{1}(t)$ is shown, where a more accurate result is obtained by the proposed control strategy in comparison with Wai (2003) and a less noisy outcome is obtained in comparison with Sawada and Itamiya (2012).

\section{Conclusion}

The design, development and implementation of an adaptive self recurrent wavelet neural network and sliding mode controller/observer for a slider crank mechanism is presented. It is proved that a full order observer for this mechanical system can be 

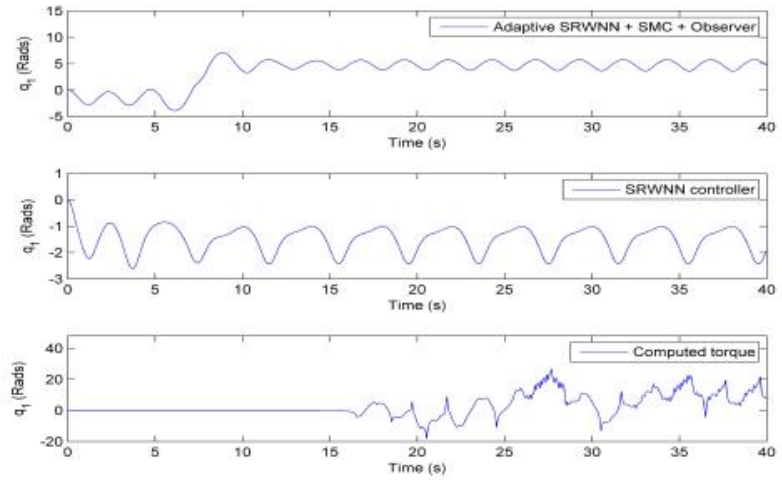

Figure 7 Angular displacement of the rotational arm $q_{1}(t)\left(\theta_{1}(t)\right)$

designed taking into account the linear displacement measurement. Then, an adaptive self recurrent wavelet neural network and sliding mode controller provide a flexible approach for the control of the slider crank mechanism. Finally, it is corroborated that this controller/observer strategy yields significantly better results in comparison with other control strategies, and more important, this control approach can be used in other applications such as robotics and mechanical systems.

\section{Appendix: Slider crank dynamic equations components}

In this section, the symmetric inertia matrix components are shown (Spong et al, 2006). The components of the inertia tensors are represented by $I x x_{i}, I y y_{i}, I z z_{i}$, where $i=1,2,3$. The masses of the rotational arm, connecting arm and linear displacement block are represented by $m_{1}, m_{2}$ and $m_{3}$ respectively. In this section only the fourth row terms (59)-(60) are shown, with the diagonal terms (61),(63) and (65), due to space limitations.

$$
\begin{aligned}
& D_{41}(q(t))=D_{42}(q(t))=D_{43}(q(t))=0, \\
& \begin{aligned}
D_{44}(q(t)) & =m_{1}+m_{2}+m_{3}, \\
D_{11}(q(t)) & =\left(m_{1}+m_{2}+m_{3}\right) \gamma(t) \\
& +\left(m_{1}+m_{2}+m_{3}\right) \eta(t) \\
& +I z z_{1}+I z z_{2},
\end{aligned}
\end{aligned}
$$$$
\gamma(t)=\left[-\sin (\delta) a-l_{2} \sin (\epsilon)-l_{c 1} \sin \left(\theta_{1}(t)\right)\right]^{2}
$$$$
\eta(t)=\left[\cos (\delta) a+l_{2} \cos (\epsilon)+l_{c 1} \cos \left(\theta_{1}(t)\right)\right]^{2},
$$

$$
\begin{aligned}
D_{22}(q(t))=\left(m_{1}+\right. & \left.m_{2}+m_{3}\right) \rho(t)+\left(m_{1}+m_{2}+m_{3}\right) \kappa(t) \\
& +I z z_{1}+I z z_{2},
\end{aligned}
$$

where

$$
\begin{aligned}
\rho(t) & =\left[-\sin (\delta) a-l_{c 2} \sin (\epsilon)\right]^{2} \\
\kappa(t) & =\left[\cos (\delta) a+l_{c 2} \cos (\epsilon)\right]^{2} \\
\delta & =\theta_{1}(t)+\theta_{2}(t)+\theta_{3}(t) \\
\epsilon & =\theta_{1}(t)+\theta_{2}(t)
\end{aligned}
$$

$$
\begin{gathered}
D_{33}(q(t))=\left(m_{1}+m_{2}+m_{3}\right) \lambda(t)+\left(m_{1}+m_{2}+m_{3}\right) \mu(t) \\
+I z z_{1}+I z z_{2},
\end{gathered}
$$

where

$$
\begin{aligned}
& \lambda(t)=\left[\sin \left(\theta_{1}(t)+\theta_{2}(t)+\theta_{3}(t)\right)\right]^{2} a^{2}, \\
& \mu(t)=\left[\cos \left(\theta_{1}(t)+\theta_{2}(t)+\theta_{3}(t)\right)\right]^{2} a^{2} .
\end{aligned}
$$

The lengths for each arm are given by $l_{1}, l_{2}$ and $l_{3}$. The center of masses of each link are $l_{c 1}, l_{c 2}$ and $l_{c 3} ; g$ is the gravity constant and the respective angular and linear velocities are given by $\theta_{i}(t)$ and $a$, respectively.

\section{References}

Abiyev R, Kaynak O, Kayacan E (2013) A type-2 fuzzy wavelet neural network for system identification and control. Journal of the Franklin Institute 350(7):16581685

Alexandridis A, Zapranis A (2013) Wavelet neural networks: A practical guide. Neural Networks 42:1-27

Azar AT, Serrano FE (2015) Adaptive sliding mode control of the Furuta pendulum. In: Advances and Applications in Sliding Mode Control Systems, Studies in Computational Intelligence

Azar AT, Serrano FE (2018) Adaptive decentralised sliding mode controller and observer for asynchronous nonlinear large-scale systems with backlash. Int J of Modelling, Identification and Control 30(1):61-71 
Azar AT, Zhu Q (2015) Advances and Applications in Sliding Mode Control systems, vol 576. Studies in Computational Intelligence, Springer

Davila J, Fridman L, Levant A (2005) Second-order sliding-mode observer for mechanical systems. IEEE Transactions on Automatic Control 50(11):1785 - 1789

Haddad M, Chellaboina V (2002) A unification between partial stability and stability theory for time-varying systems. Control Systems Magazine 22(6):66-75

Hongyan W, Qinglin W, Jihong Q, Yuhui X (2007) Wavelet neural network disturbance observer-based adaptive robust tracking control for servo system. In: Chinese Control Conference

Hongyan W, Jihong Q, Qinglin W (2010) A hybrid adaptive wavelet neural network control and sliding mode control for electro-hydraulic servo system. In: 29th Chinese Control Conference (CCC), 2010

Kalsi K, Lian J, Hui S, Zak S (2009) Sliding-mode observers for uncertain systems. In: American Control Conference, 2009. ACC' '09

Komaita Y, Furuta K (2008) Energy control of slider-crank mechanism. In: SICE Annual Conference, 2008

Lin FJ, Fung RF, Lin HH, Hong CM (1999) A supervisory fuzzy neural network controller for slidercrank mechanism. In: Proceedings of the 1999 IEEE International Conference on Control Applications

Liu M (1999) Decentralized control of robot manipulators: nonlinear and adaptive approaches. IEEE Transactions on Automatic Control 44(2):357-363

Min LC, Fu TC, Chih CC (2014) Intelligent control system design for UAV using a recurrent wavelet neural network. Neural Computing and Applications 24:487-496

Saito H, Ishikawa J, Kamamichi N, Shiotsuki T, Furuta K (2009) Self-tuning control for rotational speed of slidercrank mechanism. In: ICCAS-SICE 18-21

Sawada M, Itamiya K (2012) A position control of 2 DOF flexible link robot arms based on computed torque method. In: 2012 International Conference on Advanced Mechatronic Systems (ICAMechS)

Seraji H (1989) Decentralized adaptive control of manipulators: theory, simulation, and experimentation. IEEE Transactions on Robotics and Automation $5(2): 183-201$

Slotine J, Weiping L (1988) Adaptive manipulator control: A case study. IEEE Transactions on Automatic Control 33(11):995 - 1003

Spong MW, Hutchinson S, Vidyasagar M (2006) In: Robot Modeling and Control

Su Y, Muller P, Zheng C (2007) A simple nonlinear observer for a class of uncertain mechanical systems. IEEE Transactions on Automatic Control 52(7):1340-1345

Taha SMR, Taha ZK (2018) EEG signals classification based on autoregressive and inherently quantum recurrent neural network. Int $\mathrm{J}$ Computer applications in Technology 58(4):340351, DOI 10.1504/IJCAT.2018.095942, URL https://doi.org/10.1504/IJCAT.2018.095942

Tahmasebi R, Alizadeh H, Rahimi S, Boulet B (2014) Robust H-infinity force control of a solenoid actuator using experimental data and finite element method. In: IEEE Conference on Control Applications (CCA), 2014
Vaidyanathan S, Azar AT (2015) Anti-synchronization of identical chaotic systems using sliding mode control and an application to vaidyanathan-madhavan chaotic systems. In: Azar AT, Zhu Q (eds) Advances and Applications in Sliding Mode Control Systems, Studies in Computational Intelligence, vol 576, Springer, Berlin, Germany, pp 527-547

Vaidyanathan S, Sampath S, Azar AT (2015) Global chaos synchronisation of identical chaotic systems via novel sliding mode control method and its application to zhu system. Int $\mathrm{J}$ of Modelling, Identification and Control 23(1):92-100

Wai RJ (2003) Robust control for nonlinear motormechanism coupling system using wavelet neural network. IEEE Transactions on Systems, Man, and Cybernetics, Part B: Cybernetics 33(3):489-497

Wang J, Zong Q, Su R, Tian B (2014) Continuous high order sliding mode controller design for a flexible air-breathing hypersonic vehicle. ISA Transactions 53(3):690-698

De Wit C, Fixot N (1991) Adaptive control of robot manipulators via velocity estimated feedback. In: 1991 proceedings of the IEEE International Conference on Robotics and Automation

Wu X, Wang Y, Dang X (2014) Robust adaptive sliding-mode control of condenser-cleaning mobile manipulator using fuzzy wavelet neural network. Fuzzy Sets and Systems $235: 62-82$

Xiang C, Siow SY (2004) Control, automation, robotics and vision conference, 2004. icarcv 2004 8th. In: ICCASSICE 18-21

Xiao L, Zhu Y (2014) Sliding mode output feedback control based on tracking error observer with disturbance estimator. ISA Transactions

Yoo S, Park J, Choi Y (2007) Indirect adaptive control of nonlinear dynamic systems using self recurrent wavelet neural networks via adaptive learning rates. Information Sciences 177(15):3074-3098

Yoo SJ, Park JB, Choi YH (2005) Direct adaptive control using self recurrent wavelet neural network via adaptive learning rates for stable path tracking of mobile robots. In: Proceedings of the 2005 American Control Conference, 2005

Yoo SJ, Park JB, Choi YH (2006) Adaptive dynamic surface control of flexible-joint robots using self-recurrent wavelet neural networks. IEEE Transactions on Systems, Man, and Cybernetics, Part B: Cybernetics 36(6):1342 1355

\section{Note}

${ }^{1}$ http://www.kakadusoftware.com 